\title{
Bilateral Phrenic Nerve Block For the Treatment of Intractable Hiccup in a Palliative Care Patient: A Case Report
}

(D) Mustafa SÜREN1, id Vildan KÖLÜKÇÜ1, id Selim ADATEPE1', iD Serkan DOĞRU1, iD Ahmet AKBAŞ², id İsmail OKAN2

${ }^{1}$ Gaziosmanpaşa University Faculty of Medicine, Department of Anesthesia and Reanimation, Tokat, Turkey

${ }^{2}$ Gaziosmanpaşa University Faculty of Medicine, Department of Surgical Oncology, Tokat, Turkey

\begin{abstract}
Hiccup is the characteristic voice caused by the sudden closure of the glottis during the contraction of the muscles of respiration especially diaphragm. It usually ends spontaneously in a short time. If it lasts more than one month, it is called as intractable hiccup. Intractable hiccups may lead to malnutrition, hypoxia, arrhythmia, dehydration, depression, tiredness and sleep disorder. Those can affect the quality of life of the patient. It is reported that pharmacologic agents such as gamma-aminobutyric acid receptor agonists, dopamine antagonists, antipsychotics and baclofen may be beneficial in the treatment of intractable hiccups. If success is not achieved with these methods, invasive methods such as phrenic nerve block, regional anesthesia, and phrenic nerve pulse radiofrequency may be applied as alternative therapies. In this case report, it is aimed to present the medical management of a palliative care patient with intractable hiccup. A 55-year-old male patient underwent gastrectomy and distal esophagectomy due to esophagogastric junction tumor. He suffered from intractable hiccup after esophageal hematoma. Bilateral phrenic nerve block was performed; hence no recovery was achieved after five weeks of medical treatment. Most of his complaint about hiccup was recovered after phrenic nerve block.
\end{abstract}

Keywords: Hiccup, phrenic nerve block, esophageal diseases

\section{Introduction}

In babies in mother's womb, hiccups are frequent and are considered as physiological. This is thought to be due to the preparation of the infant's inspiratory respiratory muscles for respiration after birth. However, hiccups do not serve a physiological purpose in adults; they disturb the humans and the environment due to the sound emitted. Hiccup is a symptom accompanied by a sound which results from sudden and involuntary contractions of accessory respiratory muscles and diaphragm together with the simultaneous closure of the glottis. Hiccups are mostly benign and their frequency can range from 4 to 60 per minute and heal spontaneously in a short time (1). Hiccups are classified into 3 types: Hiccups ending up in 48 hours, persistent hiccups lasting 48 hours- 1 month and intractable hiccups lasting more than 1 month (2). Persistent hiccups can cause extremely vital problems such as malnutrition, exhaustion, fatigue, weight loss, hypoxia, bradycardia, arrhythmia, heart block, disturbed sleep patterns, speech disorders, depression, weakness, and dehydration (3). Hiccup reflex arch is composed of afferent pathway consisting of sympathetic nervous system, phrenic and vagus nerves; and efferent pathway connected to glottis and accessory respiratory muscles; and central mediators.

Cerebral events which cause deterioration in reflex arch, neckrelated diseases, esophageal, stomach and other gastrointestinal diseases, thoracic diseases such as pneumonia and tumor, cardiovascular diseases, metabolic causes such as alcohol and hypocapnia, pharmacological agents such as carboplatin and cyclophosphamide are among the causes of resistant and persistent hiccups (Table 1) $(1,4)$.

Address for Correspondence: Mustafa SÜREN, Gaziosmanpaşa University Faculty of Medicine, 
Hiccups are usually relieved with physical maneuvers, such as breathing by closing the nose, increasing carbon dioxide by holding the breath, nasopharyngeal irritation and drinking water slowly for a long time. Patients whose symptoms are not relieved by physical maneuvers can be treated mostly by noninvasive pharmacological methods such as chlorpromazine, gabapentin, metoclopramide, baclofen and proton pump inhibitors. In cases where conservative or pharmacological treatment fails, phrenic nerve block or regional anesthesia may be applied. In cases where these treatments are inadequate, studies have shown that pulsed radiofrequency to the phrenic nerve can be performed $(4,5)$.

\section{Case Report}

A 55-year-old male who was followed up in the surgical oncology service had complaints of loss of appetite, vomiting, malnutrition and insomnia due to hiccups lasting for five weeks. Four months ago, due to gastroesophageal junction tumor, total gastrectomy and distal esophagectomy were performed; two months after surgery, stent was placed due to anastomotic leak. The stent was removed back when it was determined that the stent was migrated. In the computerized tomography of the patient after severe hiccups started, hematoma in the area of anastomosis and empyema in the right lung were detected, and a chest tube was inserted to drain the empyema. The patient who did not respond to conservative treatment, including chlorpromazine, was scheduled for bilateral phrenic nerve block. After completing $5 \mathrm{~mL} 0.5 \%$ bupivacaine and $4 \mathrm{mg}$ dexamethasone to $10 \mathrm{~mL}$ with saline, this mixture was applied to both sides with $22 \mathrm{G}$ stimulator needle (Stimuplex, Braun) between sternocleidomastoid and anterior scalene muscles after contraction of diaphragma was detected (Figure 1). In the five-week follow-up of the patient after the phrenic nerve block, the patient had a 70\% decrease in hiccup frequency and severity, and consequently, the severity of the symptom cluster of loss of appetite, insomnia and fatigue was observed to be significantly reduced. After 5 weeks of phrenic nerve block, the patient's hiccups and symptoms of loss of appetite, sleeplessness and fatigue recurred, and in the computerized tomography, widespread involvement of periton and tumor recurrence at anastomosis side were observed. Bilateral phrenic nerve blocks were applied to the patient. Fourteen hours after procedure, first hiccup attack occured and lasted 10 minutes. Second hiccup attack occured 3.5 hours after first attack and lasted 15 minutes. On the $3^{\text {rd }}$ day of the procedure, 910 hiccup attacks occured and each lasted 2-3 minutes. The frequency and severity of hiccup reduced by $70 \%$ at the 6 th week of the procedure. Symptoms of malnutrition, vomiting, loss of appetite, and sleep deprivation were observed to improve nearly completely. The patient was followed by palliative care unit for 5 months after the last phrenic block. During this time, hiccups were rarely seen. The patient died in the palliative care unit. Oral and written consents were obtained from the patient during all the procedures that were planned to be performed.

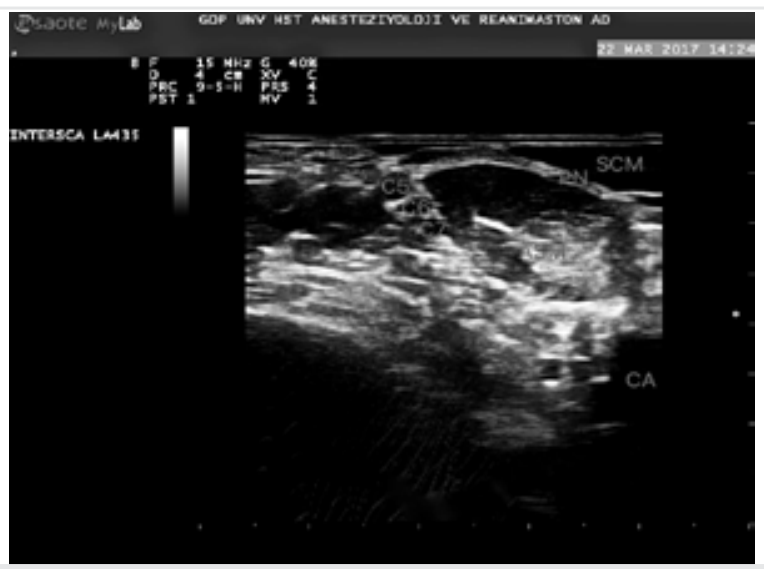

Figure 1. Ultrasound image of phrenic nerve block (SCM: Sternocleiodomastoid muscle, CA: Carotid artery, ASM: Anterior scalen muscle, PN: Phrenic nerve, C5: Fifth cervical root, C6: Sixth cervical root, C7: Seventh cervical root)

Table 1. Causes of resistant and persistent hiccups

\section{Postoperative}

Central nervous system diseases

Irritation of vagal and phrenic nerves

Gastrointestinal system diseases

Cardiovascular diseases

Thoracic diseases

Toxic-metabolic disorders

Drugs

Psychological
General anesthesia, intubation (glottic stimulation), neck extension (stretch of phrenic nerve root), pulling out of internal organs

Vascular diseases, infections, structural disturbances such as head trauma

A neck mass, goiter, pharyngitis, irritation of tympanic membrane

Gastric distention, ulcer, pancreatic disease, gastric carcinoma, abdominal abscess, inflammatory bowel disease, hepatitis, aerophagia, etc.

Myocardial infarction, pericarditis

Enlarged lymph nodes, pneumonia, empyema, bronchitis, astma, pleuritis, aortic aneurysm, mediastinitis, mediastinal tumor, chest trauma, pulmonary embolism

Alcohol, diabetes mellitus, herpes zoster, influenza, malaria, hypocalcemia, hypocapnia, hyponatremia, tuberculosis, uremia

Alpha methyl dopa, short-acting barbiturates, chemotherapy drugs (carboplatin, cisplatin), dexamethasone, diazem

Anorexia nevroza, konversiyon, excitement, pretending to be ill, stress 


\section{Discussion}

The rate of admission to hospital with a resistant and persistent hiccup without any physical pathology complaint is 55/100.000. Recurrent hiccups can be seen in $20 \%$ of patients with Parkinson's disease and in $10 \%$ of patients with gastroesophageal reflux. In general, the prevalence of hiccups in advanced cancer patients has been reported as $3.9-4.8 \%$ (1). In a case series, more than a quarter of patients with esophageal carcinoma experienced hiccups lasting more than 48 hours at least once. The treatment options of this symptom, which deteriorates the quality of life and brings many sets of symptoms, are as follows: Non-pharmacological treatments including simple physical manoeuvres in the first step, pharmacological treatments in the second step and invasive interventions in the third step. In the treatment of persistent hiccups, one of three interventional methods, phrenic nerve block, regional anesthesia, pulsed radiofrequency to phrenic nerve, can be considered as the option of treatment (6).

The pathogenesis of hiccup is complex and has not been clearly explained. As a result of stimulation of vagal, phrenic and sympathetic afferent fibers, efferent nerve fibers result in contractions of diaphragm and intercostal muscles. Neurotransmitters affecting the hiccup reflex arch and which are thought to be responsible for the pathophysiology of hiccup, are gamma-aminobutyric acid and dopamine. It is known that many dopamine antagonists such as chlorpromazine and dopamine agonists such as pramipexole and amantadine have been used in the treatment of persistent hiccups by acting on this complex reflex arch (7). Interventional applications such as phrenic nerve block, regional anesthesia and pulsed radiofrequency to phrenic nerve may be considered in cases who are unresponsive to pharmacological treatments. In addition, in the treatment of patients with resistant hiccup; decompression of the vagus nerve and surgical interventions involving the phrenic nerve can be applied. The last choice is surgical treatment involving the denervation of the phrenic nerve. This method can cause permanent nerve damage and partial paralysis in the diaphragm. For this reason, as the procedure may affect normal breathing, it should only be used as the last option (8).

Kuusniemi et al. (9) reported a 72-year-old patient who developed persistent hiccups after L4-5 laminectomy under general anesthesia. They reported that the patient did not respond to medical and conservative treatment and that the right phrenic nerve block was applied with ultrasonography on the postoperative $17^{\text {th }}$ day, and that ten minutes after the procedure hiccup disappeared and sensomotoric block developed on the right shoulder. They reported that hiccup did not recur in the follow-up and sensomotor block on right shoulder disappeared on the $3^{\text {rd }}$ day of the procedure (9). Arsanious et al. (10) reported a 60-year-old patient who underwent esophageal stent due to tracheoesophageal fistula reported hiccups and developed hiccup on the day after the procedure, unresponsive to conservative treatment. With ultrasonography, right phrenic nerve block was applied and $40 \%$ relief was achieved in the patient. Three days after the operation, left phrenic nerve block was applied and
$100 \%$ relief was achieved. Hiccups did not recur in the followup (10).

In additon, there are publications showing temporary relief due to single application of phrenic nerve block $(9,10)$. Renes et al. (8) administered local anesthetic infusion with a catheter for 24 hours with phrenic nerve block. They reported that when the infusion was stopped, the hiccup re-occured and that local anesthetic infusion was re-administered for 24 hours, and that at the end of the infusion, complete relief was observed (8). In the study of Kang and his colleagues, it was observed that temporary relief was provided by performing phrenic nerve block and that pulsed radiofrequency was applied accompanied by ultrasonography after phrenic nerve block and complete relief was achieved (5).

Hiccups lasted more than five weeks in our patient. Loss of appetite, vomiting, nutritional disorders and sleep disorders due to long-lasting hiccups were seen and the patient's quality of life was corrupted. Because the patient was unable to respond to medical treatment, phrenic nerve block, a two-sided invasive treatment method, accompanied by ultrasonography and peripheral nerve stimulator, was applied to the patient. The severity and frequency of the hiccups of the patient decreased after the block. When hiccups started again after a rest period of about 1 month, the operation was repeated again and a significant improvement was observed in the quality of life of the patient. We thought that the hiccups were due to the recurrence of the tumor and the phrenic nerve irritation.

\section{Conclusion}

Persistent hiccup is not just a peculiar annoyance that the patient has caused; it also causes additional disturbances such as malnutrition, exhaustion, fatigue, weight loss, hypoxia, bradycardia, arrhythmia, heart block, disturbed sleep patterns, speech disorders, depression, weakness and dehydration. In cases of hiccup that does not respond to pharmacological treatment, bilateral phrenic nerve block provides permanent treatment. Phrenic nerve block can be considered in treatment of hiccups in patients with terminal cancer, especially with gastrointestinal tumors.

\section{Ethics}

Informed Consent: Written informed consent was obtained from the patient.

Peer-review: Externally peer-reviewed.

\section{Authorship Contributions}

Concept: MS., Design: S.D., Data Collection or Processing: A.A., Analysis or Interpretation: İ.O., Literature Search: M.S., Writing: V.K.

Conflict of Interest: No conflict of interest was declared by the authors.

Financial Disclosure: The authors declared that this study received no financial support. 


\section{References}

1. Steger M, Schneemann M, Fox M. Systemic review: the pathogenesis and pharmacological treatment of hiccups. Aliment Pharmacol Ther 2015;42:1037-50.

2. Marinella MA. Diagnosis and management of hiccups in the patient with advanced cancer. J Support Oncol 2009;7:122-7.

3. Lewis JH. Hiccups: causes and cures. J Clin Gastroenterol 1985;7:539-52.

4. Bagheri H, Cismondo S, Montastruc JL. Drug-induced hiccup: a review of the France pharmacologic vigilance database. Therapie 1999;54:35-9.

5. Kang KN, Park IK, Suh JH, Leem JG, Shin JW. Ultrasound-guided pulsed radiofrequency lesioning of the phrenic nerve in a patient with intractable hiccup. Korean J Pain 2010;23:198-201.
6. Campbell P, Janak S, Hilas O. Gabapentin for the treatment of persistent hiccups. Consult Pharm 2014;29:408-12.

7. Vaidya V. Sertraline in the Treatment of Hiccups. Psychosomatics 2000;41:353-5

8. Renes SH, van Geffen GJ, Rettig HC, Gielen MJ, Scheffer GJ. Ultrasound-guided continuous phrenic nerve block for persistent hiccups. Reg Anesth Pain Med 2010;35:455-7.

9. Kuusniemi K, Pyylampi V. Phrenic nerve block with ultrasoundguidance for treatment of hiccups: a case report. J Med Case Rep 2011;5:493.

10. Arsanious D, Khoury S, Martinez E, Nawras A, Filatoff G, Ajabnoor $\mathrm{H}$ et al. Ultrasound-Guided Phrenic Nerve Block for Intractable Hiccups following Placement of Esophageal Stent for Esophageal Squamous Cell Carcinoma. Pain Physician 2016;19:E653-6. 